\title{
BAY 87-2243 sensitizes hepatocellular carcinoma Hep3B cells to histone deacetylase inhibitors treatment via GSK-3 $\beta$ activation
}

\author{
YANG-LING LI ${ }^{1,2^{*}}$, MING-JUN RAO ${ }^{1-3^{*}}$, NING-YU ZHANG ${ }^{4}$, \\ LIN-WEN WU ${ }^{5}$, NENG-MING LIN ${ }^{1-3}$ and CHONG ZHANG ${ }^{4}$ \\ ${ }^{1}$ Department of Clinical Pharmacology, Hangzhou First People's Hospital, Zhejiang Chinese Medical University; \\ ${ }^{2}$ Department of Clinical Pharmacology, Affiliated Hangzhou First People's Hospital, Zhejiang University School of \\ Medicine, Hangzhou, Zhejiang 310006; ${ }^{3}$ Institute of Pharmacology, College of Pharmaceutical Sciences, \\ Zhejiang Chinese Medical University, Hangzhou, Zhejiang 311402; ${ }^{4}$ School of Medicine, Zhejiang University City College, \\ Hangzhou, Zhejiang 310015; ${ }^{5}$ College of Pharmaceutical Sciences, Zhejiang University, \\ Hangzhou, Zhejiang 310058, P.R. China
}

Received October 2, 2018; Accepted March 12, 2019

DOI: $10.3892 /$ etm. 2019.7500

\begin{abstract}
Hepatocellular carcinoma (HCC) is associated with some of the highest cancer-associated mortality rates. Histone deacetylase (HDAC) inhibitors anti-HCC activities have been shown to promote Snail-induced metastasis. In the present study, it was shown that BAY 87-2243, a hypoxia-inducible transcription factor- $1 \alpha$ inhibitor, could enhance the anti-HCC effects of HDAC inhibitors, including trichostatin $\mathrm{A}$ and vorinostat. In addition, BAY 87-2243 plus HDAC inhibitors exhibited synergistic cytotoxicity and induced significant cell death in Hep3B cells. Additionally, BAY 87-2243 combined with HDAC inhibitors-treated Hep3B cells formed fewer and smaller colonies as compared with either the control or single agent-treated cells. Furthermore, glycogen synthase kinase- $3 \beta$ might be involved in the enhanced cell death induced by BAY 87-2243 plus HDAC inhibitors. The present data also indicated that BAY 87-2243 combined with HDAC inhibitors could suppress the migration of Hep3B cells, and BAY 87-2243 could reverse the HDAC inhibitor-induced Snail activation in Hep3B cells. In conclusion, BAY 87-2243 combined with
\end{abstract}

Correspondence to: Professor Neng-Ming Lin, Department of Clinical Pharmacology, Hangzhou First People's Hospital, Zhejiang Chinese Medical University, 261 Huansha Road, Hangzhou, Zhejiang 310006, P.R. China

E-mail:1nm1013@163.com

Dr Chong Zhang, School of Medicine, Zhejiang University City College, 51 Huzhou Street, Hangzhou, Zhejiang 310015, P.R. China

E-mail: zhangchong@zucc.edu.cn

${ }^{*}$ Contributed equally

Key words: BAY87-2243, hepatocellular carcinoma, histone deacetylase inhibitors, trichostatin A, vorinostat, glycogen synthase kinase $3 \beta$
HDAC inhibitors might be an attractive chemotherapy strategy for HCC therapy.

\section{Introduction}

Hepatocellular carcinoma (HCC), one of the most deadly cancers worldwide, accounts for the majority of primary liver cancer cases (1). The overexpression of histone deacetylases (HDACs) plays an important role in almost all cancerous behaviors of HCC, including cancer initiation, progression and chemotherapy-resistance (2). A number of HDAC inhibitors, such as vorinostat and trichostatin A, have been tested to treat HCC both in preclinical models and clinical trials (3-5). HDAC inhibitors exert their anti-tumor activities by inducing cell cycle arrest, apoptosis, autophagy and differentiation (6). Furthermore, combining HDAC inhibitors with other agents has been identified as a potential approach to overcoming chemotherapeutic resistance $(7,8)$.

Hypoxia activates genetic programs that facilitate HCC cell metastasis, proliferation, as well as chemotherapy and radiotherapy-resistance (9). Hypoxia-inducible transcription factor (HIF) is activated in HCC cells under hypoxic conditions, leading to angiogenesis and poor prognosis (10). BAY 87-2243 inhibits HIF- $1 \alpha$ and HIF-2 $\alpha$ protein accumulation under hypoxia, and BAY 87-2243 also suppresses the activity of mitochondrial complex I in non-small cell lung cancer (11). Furthermore, BAY 87-2243 significantly reduces tumor growth in BRAF mutant melanoma cancer cells by targeting mitochondrial complex I (12). BAY 87-2243 induces mitochondrial permeability transition pore opening, stimulates autophagosome formation and leads to the activation of necroptotic/ferroptotic cell death in melanoma cells (13). In addition, the reduction of tumor hypoxia by application of BAY-87-2243 before the beginning of fractionated radiotherapy can improve local tumor control (14).

The activation of HDAC and hypoxia are associated with cancer initiation, progression and chemo-resistance in HCC cells $(9,10)$. The current study aimed to investigate whether 
blocking both HDAC and hypoxia can be efficient to treat HCC. Thus, the authors hypothesized that combining HIF-1 $\alpha$ inhibitor with HDAC inhibitor could be a logical combination regimen to treat $\mathrm{HCC}$.

\section{Materials and methods}

Cell culture and reagents. BAY 87-2243 (cat. no. S7309), CHIR-99021 (cat. no. S2924), vorinostat (cat. no. S1047) and trichostatin A (cat. no. S1045) were purchased from Selleck Chemicals. Hep3B cells were purchased from the Cell Bank of the Chinese Academy of Sciences. The cells were cultured in MEM medium containing $10 \%$ fetal bovine serum (PAN Biotech) at $37^{\circ} \mathrm{C}$ in a humidified atmosphere with $5 \% \mathrm{CO}_{2}$. Propidium iodide (PI; cat. no. 70-ZF-50-0001) solution was purchased from Multi-Sciences Biotech.

Cell proliferation assays. Hep3B cells were seeded into 96-well plates at a density of $4 \times 10^{3}$ per well and were allowed to grow overnight at $37^{\circ} \mathrm{C}$. The cells were incubated with BAY 87-2243 (2 or $4 \mu \mathrm{M})$ and/or HDAC inhibitors $(0.2-4 \mu \mathrm{M})$ for $72 \mathrm{~h}$ at $37^{\circ} \mathrm{C}$. Cell proliferation was detected by the SRB assay, as previously described (9). Briefly, cells were fixed with $10 \%$ trichoroacetic acid solution at $4^{\circ} \mathrm{C}$ overnight. Subsequent to washing, $0.4 \%$ SRB solution (100 $\mu 1$ per well; Sigma-Aldrich) was added into each well. After $20 \mathrm{~min}$ staining at room temperature, wells were rinsed with $1 \%$ acetic acid to remove unbound dye and then left to air dry. Subsequently, $100 \mu 1$ Tris-base lye (10 mM; NeoFROXX GmbH) was added, followed by $10-\mathrm{min}$ oscillation. The absorbance was then recorded at $515 \mathrm{~nm}$ using a multiscan spectrum.

Colony formation assay. Hep3B cells were seeded onto 6-well plates at a density of 1,000 cells per well. After 14 days, cells were stained with $0.1 \%$ crystal violet solution for $30 \mathrm{~min}$ at $4^{\circ} \mathrm{C}$ and the colonies were imaged by a camera.

Detection of cell death. Hep3B cells were seeded into 6 well plates and incubated with BAY 87-2243 (10 $\mu \mathrm{M}$; higher than the SRB assay using 96 well plates) and/or and/or HDAC inhibitors $(0.25-2 \mu \mathrm{M})$, and PI staining was then used to detect cell death, as previously described (9). Sub-G1 analysis following PI staining was used to detect apoptosis. Cells were harvested, washed with PBS three times and fixed with pre-cooled $70 \%$ ethanol at $-20^{\circ} \mathrm{C}$ overnight. Cells were washed and resuspended in $500 \mu \mathrm{l}$ PBS containing $50 \mu \mathrm{g} / \mathrm{ml}$ RNase at $37^{\circ} \mathrm{C}$ for $30 \mathrm{~min}$. The cells were then stained with $5 \mu \mathrm{g}$ PI at room temperature for $30 \mathrm{~min}$. For each sample, $2 \times 10^{4}$ cells were collected and analyzed using a FACS-Calibur cytometer and the data were analyzed using Cellquest Software (version 6.0; BD Biosciences).

Western blot analysis. Western blot analysis was performed as previously described (9). Proteins were extracted with lysis buffer containing $150 \mathrm{mM} \mathrm{NaCl}, 50 \mathrm{mM}$ Tris- $\mathrm{HCl}, 0.1 \%$ sodium dodecyl sulfate, $1 \mathrm{mM}$ ethylenediaminetetraacetic acid, $0.5 \%$ deoxycholic acid, 1\% NP-40, $2.0 \mu \mathrm{g} / \mathrm{ml}$ aprotinin, $1 \mathrm{mM}$ phenylmethylsulfonylfluoride and $0.02 \%$ sodium azide (Beyotime Institute of Biotechnology). The lysates were centrifuged at $10,000 \mathrm{x} \mathrm{g}$ for $30 \mathrm{~min}$ at $4^{\circ} \mathrm{C}$, then the concentrations of protein were determined by the BCA method using Enhanced BCA Protein Assay kit (Beyotime Institute of Biotechnology). Total proteins (40 $\mu \mathrm{g} /$ lane) were fractionated on $8-15 \%$ Tris-glycine gels, and then they were transferred to polyvinylidene fluoride membrane, blocked with 5\% (w/v) Difco Skim milk (cat. no. 232100; BD Biosciences) in PBS at room temperature for $1 \mathrm{~h}$ and probed with the primary antibodies at $4^{\circ} \mathrm{C}$ overnight. The proteins were incubated and visualized with horseradish peroxidase-conjugated goat anti-mouse (cat. no. GAM007) and anti-rabbit (cat. no. GAM007) IgG secondary antibodies (MultiSciences) at a dilution of 1:5,000 for $1 \mathrm{~h}$ at room temperature. Finally, proteins were visualized using the enhanced chemiluminescence detection system (PerkinElmer). Anti-poly (ADP-ribose) polymerase (PARP; cat. no. sc-7150; 1:1,000), anti-AKT (cat. no. sc-135829; 1:500), anti-phospho-AKT (Ser-473; cat. no. sc-7985; 1:500) and anti-GAPDH (cat.no.sc-25778; 1:1,000) antibodies were obtained from Santa Cruz Biotechnology, Inc. Anti-phospho-glycogen synthase kinase (GSK)-3 $\beta$ (Ser-9; cat. no. 5558S; 1:500) and anti-snail antibodies (cat. no. 3879S; 1:1,000) were purchased from Cell Signaling Technology, Inc. Anti-GSK3 $\beta$ antibody (cat. no. $610202 ; 1: 1,000)$ was obtained from BD Biosciences.

Wound healing assay. Hep3B cells were seeded in 24-well plates and cultured until they reached $90 \%$ confluency. Confluent monolayer cells were gently scratched with a sterile pipette tip and then washed three times with PBS to clear cell debris and suspended cells. Fresh serum-free medium was added, and the cells were allowed to close the wound for $24 \mathrm{~h}$ under normal conditions at $37^{\circ} \mathrm{C}$. Images of the wound in the same relative position were captured with a computer-assisted microscope (model, CKX41SF; Olympus Corporation).

Statistical analysis. One-way analysis of variance followed by Tukey post hoc test was used to examine the significance of differences among groups. The results were presented as the mean \pm standard deviation from three independent experiments and $\mathrm{P}<0.05$ was used to indicate a statistically significant difference.

\section{Results}

BAY 87-2243 enhances the HDAC inhibitor-induced antiproliferative effect of Hep $3 B$ cells. First, the morphological changes induced by the combined treatment of BAY 87-2243 and HDAC inhibitors was detected in Hep3B cells. Fig. 1A showed that a very large proportion of Hep3B cells treated with BAY 87-2243 plus HDAC inhibitors (trichostatin A or vorinostat) had dislodged from the dishes whereas the remaining adherent cells showed loss of adhesion, shrinkage and rounding, which are typical morphological changes associated with apoptosis (15). Furthermore, the results presented in Fig. 1B showed that BAY 87-2243 combined with HDAC inhibitors (trichostatin A or vorinostat) significantly decreased the proliferation ability of Hep3B cells following incubation for $72 \mathrm{~h}$, as compared with single agent-treatment. To further confirm the enhanced antiproliferative effect of the combined treatment with BAY 87-2243 and HDAC inhibitors, whether the combination might inhibit colony formation in Hep3B cells or not, was determined next. It was found that BAY 87-2243 combined with HDAC inhibitors-treated Hep3B cells formed 
A

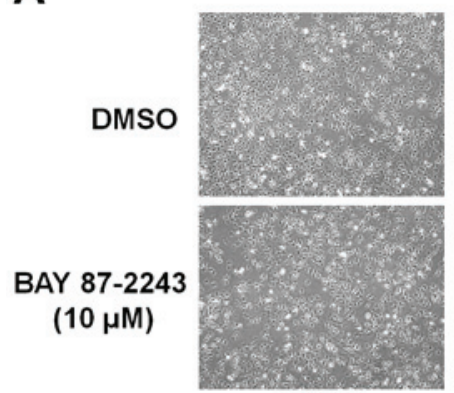

DMSO
Hep3B

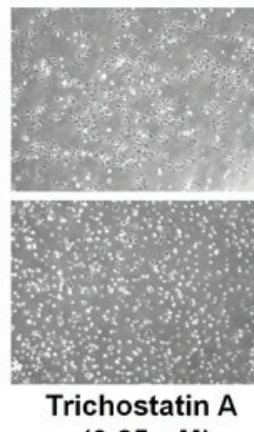

$(0.25 \mu \mathrm{M})$
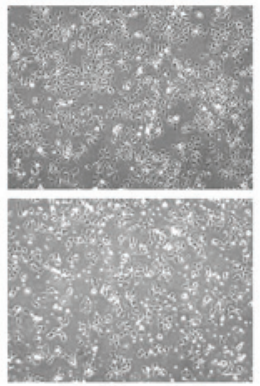

Vorinostat

$(2 \mu \mathrm{M})$

B

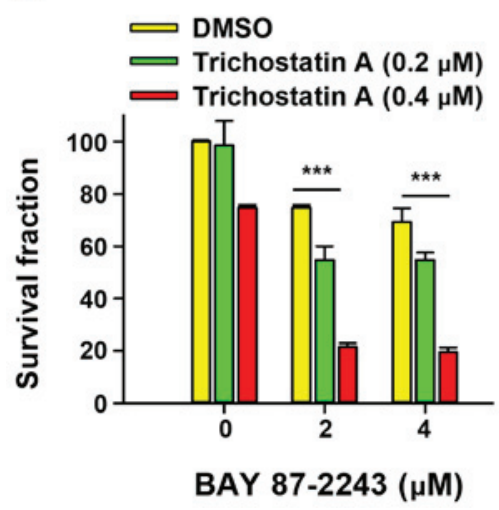

Нер3B

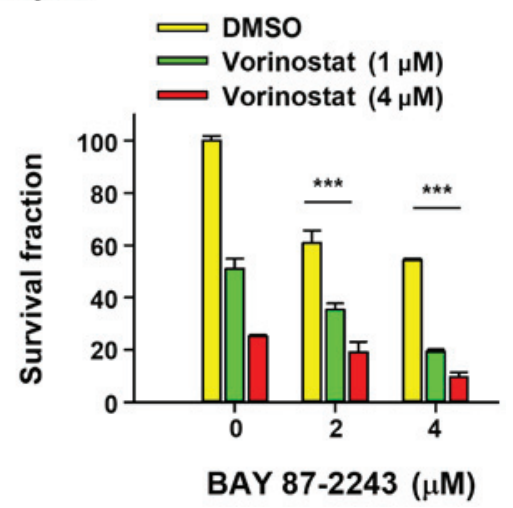

Figure 1. BAY 87-2243 enhances the anti-proliferation effect of Hep3B cells induced by HDAC inhibitors. (A) Hep3B cells were plated in 6-well plates and treated with BAY 87-2243 and/or HDAC inhibitors at the indicated concentrations for $72 \mathrm{~h}$, and cell morphological changes were observed. Scale bar, $20 \mu \mathrm{m}$. (B) Hep3B cells were plated in 96-well plates and incubated with the compounds for $72 \mathrm{~h}$. SRB assay was used to detect the proliferation of Hep3B cells. ${ }^{* * *} \mathrm{P}<0.001$. HDAC, histone deacetylase.

A

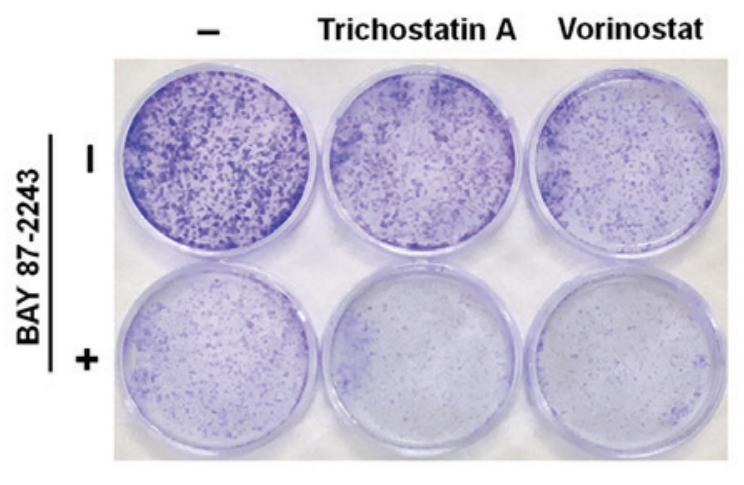

B

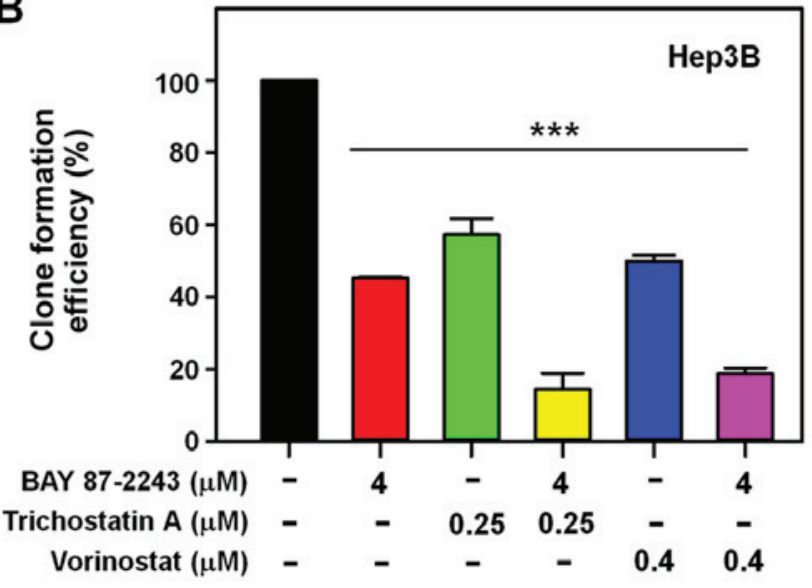

Figure 2. BAY 87-2243 enhances the anti-proliferation effects of Hep3B cells induced by HDAC inhibitors in colony formation assay. (A) Hep3B cells were treated with BAY 87-2243 $(4 \mu \mathrm{M})$ and/or HDAC inhibitors (Trichostatin A, $0.25 \mu \mathrm{M}$; Vorinostat, $0.4 \mu \mathrm{M}$ ) for 14 days. (B) BAY 87-2243 enhanced the anti-proliferation effects of Hep3B cells induced by HDAC inhibitors in colony formation assay. ${ }^{* * *} \mathrm{P}<0.001$. HDAC, histone deacetylase.

fewer and smaller colonies as compared with either the control or single agent-groups $(\mathrm{P}<0.0001$; Fig. $2 \mathrm{~A}$ and $\mathrm{B})$. These results confirmed that BAY 87-2243 enhanced the HDAC inhibitor-induced antiproliferative effect in Hep3B cells.

BAY 87-2243 enhances HDAC inhibitor-induced cell death in Hep3B cells. To determine whether the enhanced antiproliferative effect of BAY 87-2243 plus HDAC inhibitors resulted from cell death, PI staining was used to detect the cell death in Hep3B cells treated with BAY 87-2243 and/or HDAC inhibitors. The rate of cell death in Hep3B cells treated with BAY 87-2243 and trichostatin A was $70.13 \%$, and it was significantly higher than that in the BAY 87-2243 group (28.94\%) and trichostatin A group only (24.39\%) following treatment for $48 \mathrm{~h}$ (Fig. 3A; upper panel). Similarly, the combination treatment of BAY 87-2243 and vorinostat 
A
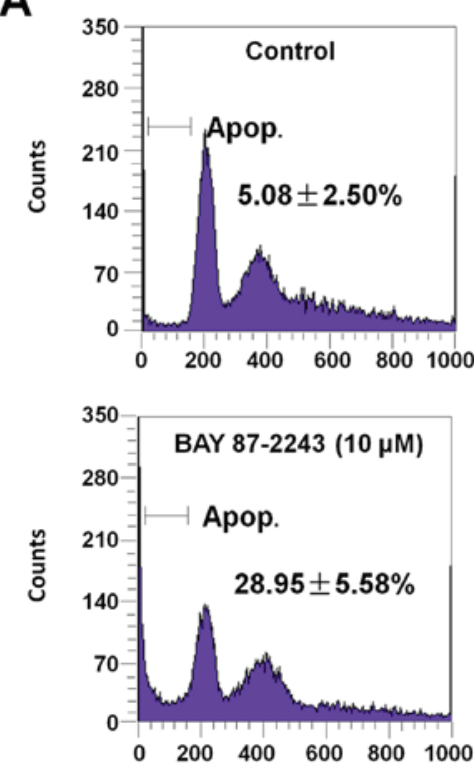

Hep3B
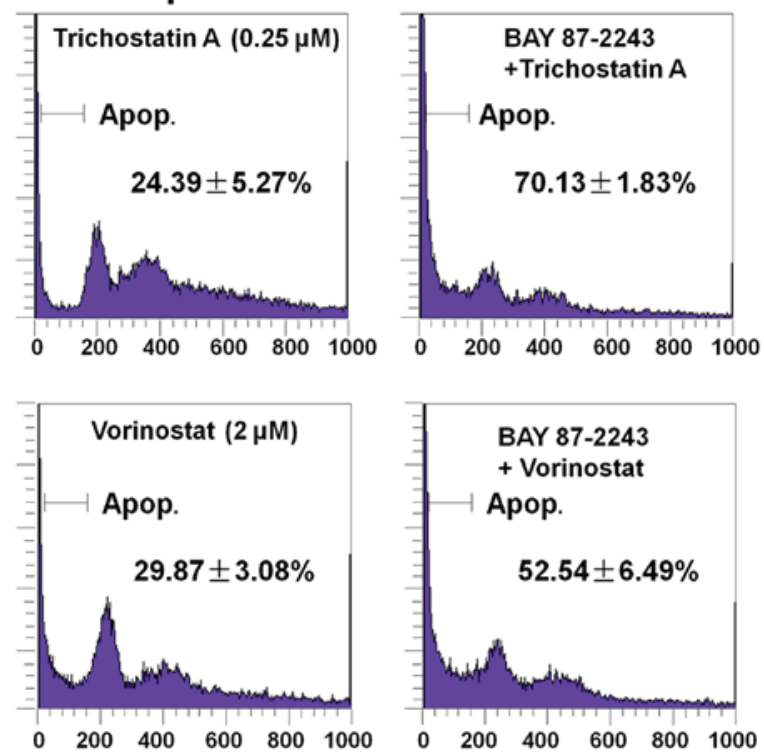

Propidium iodide

B

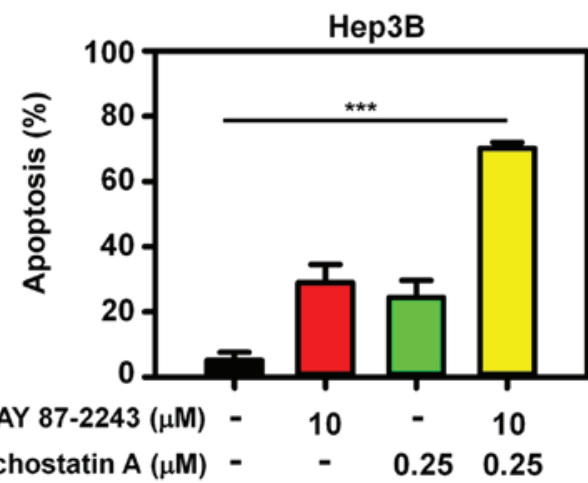

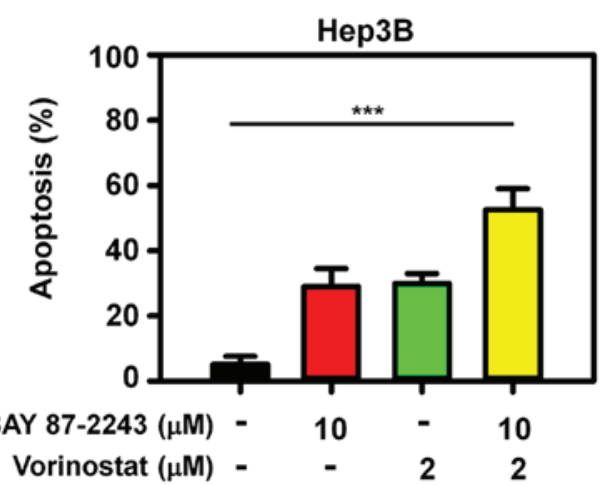

Figure 3. BAY 87-2243 promotes HDAC inhibitors-induced cell death in Hep3B cells. (A) Hep3B cells were plated into 6-well plates and incubated with BAY 87-2243 $(10 \mu \mathrm{M})$ and/or HDAC inhibitors for $48 \mathrm{~h}$, and then cells were incubated with propidium iodide and were analyzed by flow cytometry, to detect the cells in the sub-G1 phase. (B) BAY 87-2243 promoted HDAC inhibitors-induced cell death in Hep3B cells. ${ }^{* * *}$ P<0.001. HDAC, histone deacetylase.

resulted in an increased apoptotic rate, as compared with single agent groups (Fig. 3A; lower panel). Therefore, BAY 87-2243 enhanced HDAC inhibitor-induced cell death in Hep3B cells $(\mathrm{P}<0.0001$; Fig. 3B). Furthermore, the BAY 87-2243 plus HDAC inhibitor-induced enhanced apoptosis was accompanied by higher expression of the cleaved PARP, in Hep3B cells (Fig. 4A).

BAY 87-2243 combined with HDAC inhibitors induces cell death via GSK3 $\beta$ activation. The AKT/GSK-3 $\beta /$ Snail pathway has been shown to be involved in HCC metastasis by inducing epithelial-mesenchymal transition (EMT) $(16,17)$. The transcription factor Snail, controls EMT by repressing the expressions of E-cadherin and other epithelial genes, and GSK-3 $\beta$ phosphorylates Snail inducing its degradation (18). The present data indicated that BAY 87-2243 plus HDAC inhibitors could markedly inhibit AKT, Snail and activate GSK-3 $\beta$ by suppressing phosphorylated GSK-3 $\beta$ (Ser-9; Fig. 4A). Furthermore, it was found that GSK-3 $\beta$ inhibitor CHIR-99021 could partially reverse the cell death induced by BAY 87-2243 plus HDAC inhibitors (Fig. 4B; P<0.001). Thus, the potential ability of BAY 87-2243 plus HDAC inhibitors to inhibit the AKT/GSK-3 $\beta /$ Snail pathway makes it an attractive chemotherapy strategy to hinder HCC metastasis, but this needs to be investigated further.

BAY 87-2243 combined with HDAC inhibitors suppresses the migration of Hep3B cells. As shown in Fig. 5, untreated Hep3B cells migrated within $24 \mathrm{~h}$ after wounding, whereas there was moderate inhibition of cell migration when the Hep3B cells were treated with BAY 87-2243 $(10 \mu \mathrm{M})$ or Trichostatin A $(0.25 \mu \mathrm{M})$. However, BAY 87-2243 plus Trichostatin A could significantly suppress the migration of Hep3B cells $(\mathrm{P}<0.001)$. Similarly, BAY 87-2243 plus Vorinostat could also significantly inhibit the migration of Hep3B cells.

\section{Discussion}

$\mathrm{HCC}$ is a metabolically heterogeneous cancer, and the use of glucose by HCC cells can influence their tumorigenicity (19). Therefore, increased glucose metabolism is important for the growth of HCC cells (20). In addition, the suppression of 
A

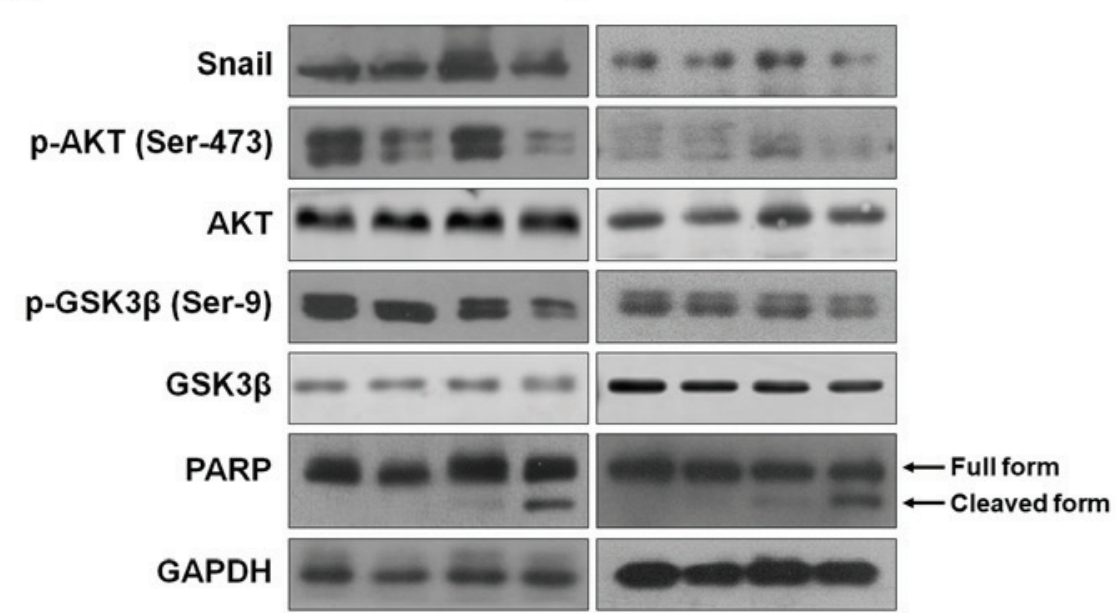

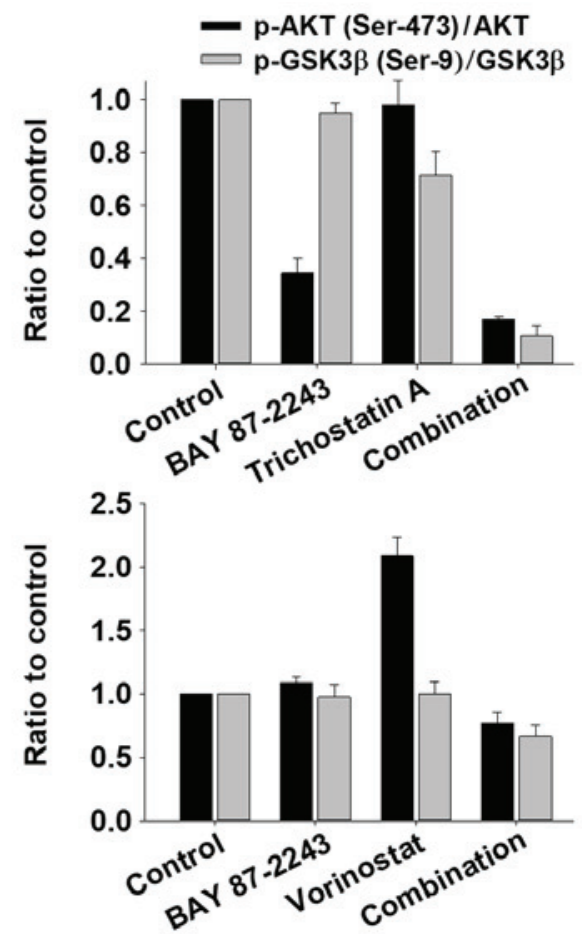

B
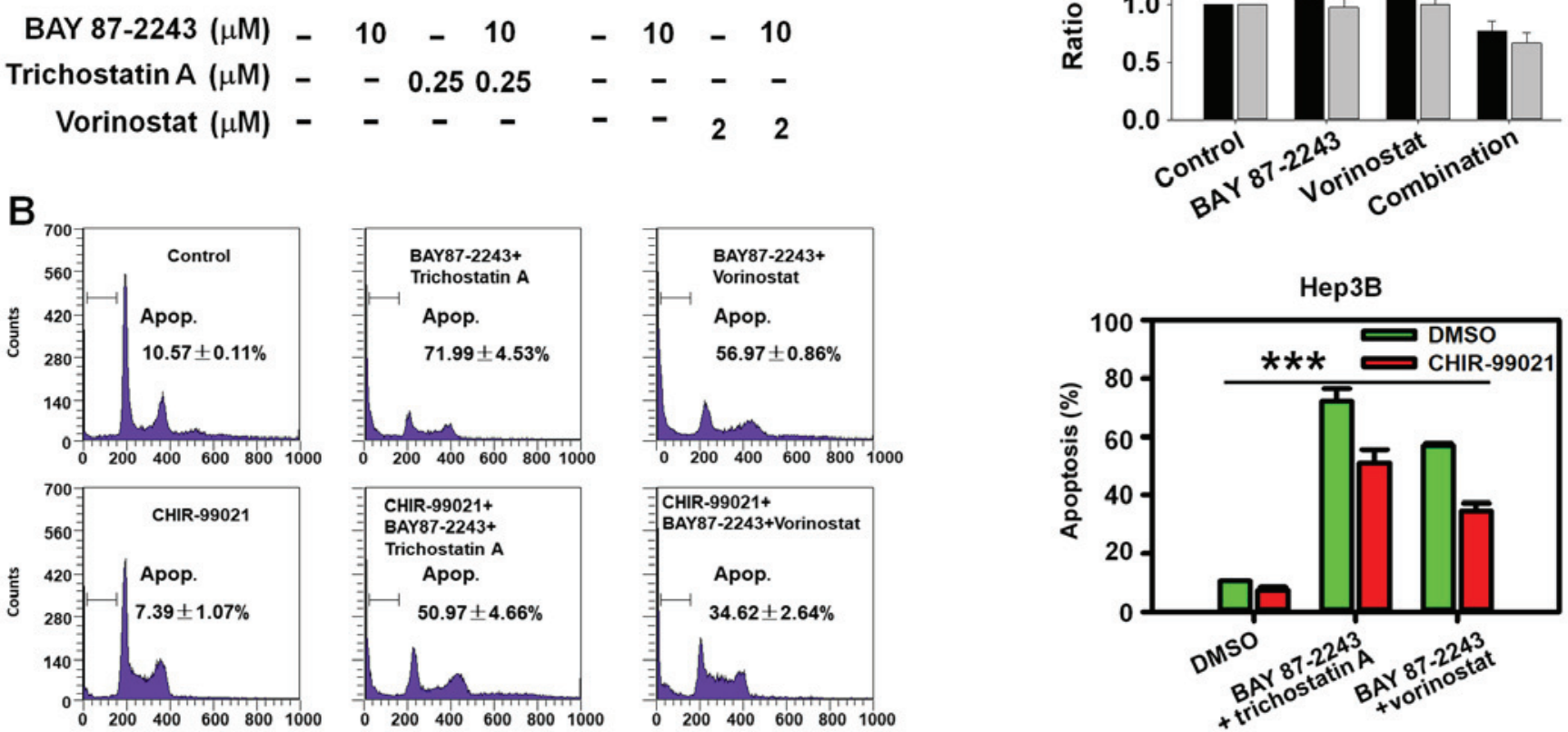

Figure 4. GSK-3 $\beta$ is involved in BAY 87-2243 plus HDAC inhibitors treatment-induced cell death. (A) Hep3B cells were treated with compounds at indicated concentrations for $48 \mathrm{~h}$, after which the expression of proteins was detected by western blotting. (B) Hep3B cells were pre-treated with CHIR-99021 ( $5 \mu \mathrm{M}$ ) for $1 \mathrm{~h}$, and then treated with BAY 87-2243 $(10 \mu \mathrm{M})$ and/or HDAC inhibitors (Trichostatin A, $0.25 \mu \mathrm{M}$; Vorinostat, $2 \mu \mathrm{M})$ for $48 \mathrm{~h}$. Propidium iodide staining was used to detect sub-G1 phase cells. ${ }^{* * *} \mathrm{P}<0.001$. GSK, glycogen synthase kinase; HDAC, Histone deacetylase; p, phosphorylated; PARP, poly (ADP-ribose) polymerase.
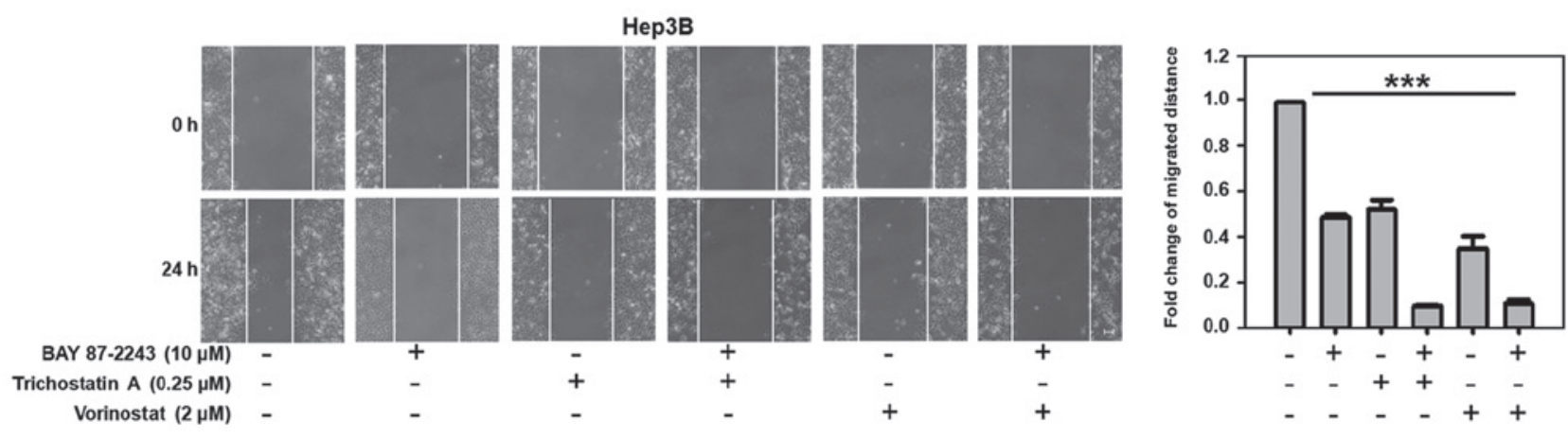

Figure 5. BAY 87-2243 plus HDAC inhibitors suppress the migration of hepatocellular carcinoma cells. Hep3B cells were gently scratched with a sterile pipette tip and then washed with PBS three times. Subsequently, Hep3B cells were incubated with the compounds at the indicated concentrations for $24 \mathrm{~h}$. The quantification of the fold change of average migrated distance $(24 / 0 \mathrm{~h})$ was shown in a wound healing assay. Scale bars, $20 \mu \mathrm{m} .{ }^{*} *{ }^{2}<0.001$. HDAC, histone deacetylase.

HDACs inhibits glucose metabolism and HCC cell growth by restoring fructose-1,6-bisphosphatase activity (3). BAY
87-2243 inhibits cell proliferation with low efficacy under standard conditions, but high efficacy under glucose depletion, 


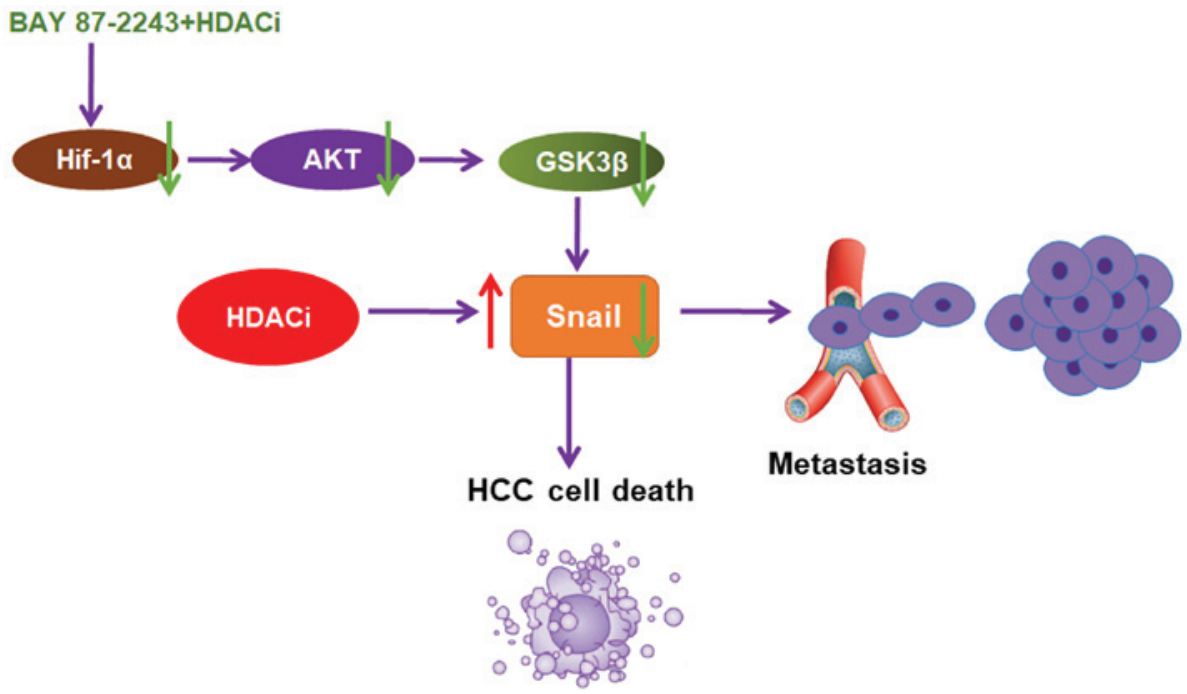

Figure 6. GSK-3 $\beta /$ Snail pathway is involved in the synergistic effect of BAY 87-2243 plus HDAC inhibitors. HDAC inhibitors can activate Snail and induce metastasis of HCC cells. BAY 87-2243, as a HIF-1 $\alpha$ inhibitor, may reverse HDAC inhibitors-initiated metastasis in HCC cells via AKT/GSK-3//Snail signaling pathway.

a condition favoring mitochondrial generation as an energy source (11). In the present study, it was hypothesized that HDAC inhibitors might increase the anti-HCC efficacy of BAY 87-2243 in HCC cells. Indeed, the results demonstrated that BAY 87-2243 may suppress the proliferation of HCC cells, and BAY 87-2243 plus HDAC inhibitors (trichostatin A or vorinostat) could distinctly inhibit proliferation and induce cell death in Hep3B cells. Therefore, a combination chemotherapy regimen incorporating BAY 87-2243 and HDAC inhibitors may be an effective chemotherapy strategy for the treatment of HCC.

HIF- $1 \alpha$ could be activated by hypoxia-independent mechanisms such as the PI3K/AKT/mTOR signaling pathway (21). In the present study, BAY 87-2243 as a HIF-1 $\alpha$ inhibitor could decrease $\mathrm{p}-\mathrm{AKT}$ (ser-473), indicating that HIF-1 $\alpha$ suppression could also affect PI3K/AKT/mTOR signaling pathway. Furthermore, the present data also indicated that BAY 87-2243 plus HDAC inhibitors could suppress PI3K/AKT/mTOR signaling. In addition, the inactivation of GSK-3 $\beta$ plays an important role in mediating the improved glucose metabolism, and GSK-3 $\beta$ (Ser-9) phosphorylation reduces the activity of GSK-3 $\beta(22,23)$. In the present study, it was shown that BAY 87-2243 plus HDAC inhibitors could activate GSK-3 $\beta$ by suppressing phosphorylated GSK-3 $\beta$ (Ser-9). In addition, GSK-3 $\beta$ inhibitor CHIR-99021 successfully reversed the cell death induced by BAY 87-2243 plus HDAC inhibitors. These data indicated that BAY 87-2243 plus HDAC inhibitors might suppress glucose metabolism by activating GSK-3 $\beta$ in HCC cells.

The majority of HCC patients are diagnosed in advanced stages of the disease, and the poor survival in patients with $\mathrm{HCC}$ can be largely attributed to rapid intrahepatic recurrence due to cancer metastases (24). HDAC inhibitors, including trichostatin $\mathrm{A}$ and vorinostat, induce cell death but simultaneously augment cell migration and metastasis to ruin therapeutic efficacy via activating protein kinase $\mathrm{C}$ signaling (25). Furthermore, HDAC inhibitors are able to promote the expression of Snail, and trigger Snail-induced EMT which is critical for HDAC inhibitors-initiated invasion and metastasis (26). The findings of the present study highlighted that BAY 87-2243 could reverse the HDAC inhibitor-induced activation of Snail in HCC cells. Furthermore, the present data showed that BAY 87-2243 plus HDAC inhibitor could significantly suppress the migration of HCC cells, indicating that combining BAY 87-2243 with HDAC inhibitors appears to be an attractive option for the patients with metastatic HCC. However, further research is required to elucidate the anti-metastatic activity of BAY 87-2243 plus HDAC inhibitors in HCC.

In conclusion, the present data provided, to the best of our knowledge, the first evidence that BAY 87-2243 could enhance the anti-HCC effect of HDAC inhibitors through the AKT/GSK-3 $\beta /$ Snail signaling pathway (Fig. 6). It was also shown that BAY 87-2243 plus Vorinostat could inhibit the migration of Hep3B cells. BAY 87-2243 plus HDAC inhibitors might be an attractive chemotherapy strategy for HCC therapy.

\section{Acknowledgements}

Not applicable.

\section{Funding}

The present study was supported by Zhejiang Province Medical Key Discipline Construction (grant no. 2018-2-03, 2018), National Natural Science Foundation of China (grant no. 81702887, 2017), Hangzhou Major Science and Technology Project (grant no. 20172016A01, 2017), Fund of Hangzhou Medical Key Discipline Construction (grant no. 2017-51-07, 2017), and Zhejiang Provincial Foundation of Natural Science (grant nos. LQ16H310004, 2017 and LY19H310004, 2019).

\section{Availability of data and materials}

The analyzed data sets generated during the study are available from the corresponding author on reasonable request. 


\section{Authors' contributions}

YL, MJR, NYZ and LWW performed the experiments. NML and $\mathrm{CZ}$ conceived and designed the study. $\mathrm{CZ}$ wrote the manuscript. All authors read and approved the manuscript.

\section{Ethics approval and consent to participate}

This article does not contain any studies with animal or human participants performed by any of the authors.

\section{Patient consent for publication}

Not applicable.

\section{Competing interests}

The authors declare that they have no competing interests.

\section{References}

1. Geng Y, Michowski W, Chick JM, Wang YE, Jecrois ME, Sweeney KE, Liu L, Han RC, Ke N, Zagozdzon A, et al: Kinase-independent function of E-type cyclins in liver cancer. Proc Natl Acad Sci USA 115: 1015-1020, 2018.

2. Fu M, Shi W, Li Z and Liu H: Activation of mPTP-dependent mitochondrial apoptosis pathway by a novel pan HDAC inhibitor resminostat in hepatocellular carcinoma cells. Biochem Biophys Res Commun 477: 527-533, 2016.

3. Yang J, Jin X, Yan Y, Shao Y, Pan Y, Roberts LR, Zhang J, Huang $\mathrm{H}$ and Jiang J: Inhibiting histone deacetylases suppresses glucose metabolism and hepatocellular carcinoma growth by restoring FBP1 expression. Sci Rep 7: 43864, 2017.

4. Chen T, Gu C, Xue C, Yang T, Zhong Y, Liu S, Nie Y and Yang H: LncRNA-uc002mbe. 2 interacting with hnRNPA2B1 Mediates AKT deactivation and p21 Up-regulation induced by trichostatin in liver cancer cells. Front Pharmacol 8: 669, 2017.

5. West AC and Johnstone RW: New and emerging HDAC inhibitors for cancer treatment. J Clin Invest 124: 30-39, 2014.

6. Zhang J and Zhong Q: Histone deacetylase inhibitors and cell death. Cell Mol Life Sci 71: 3885-3901, 2014.

7. Yuan H, Li AJ, Ma SL, Cui LJ, Wu B, Yin L and Wu MC: Inhibition of autophagy signi fi cantly enhances combination therapy with sorafenib and HDAC inhibitors for human hepatoma cells. World J Gastroenterol 20: 4953-4962, 2014.

8. Yoon CY, Park MJ, Lee JS, Lee SC, Oh JJ, Park H, Chung CW, Abdullajanov MM, Jeong SJ, Hong SK, et al: The histone deacetylase inhibitor trichostatin A synergistically resensitizes a cisplatin resistant human bladder cancer cell line. J Urol 185: 1102-1111, 2011.

9. Li YL, Zhang NY, Hu X, Chen JL, Rao MJ, Wu LW, Li QY, Zhang B, Yan W and Zhang C: Evodiamine induces apoptosis and promotes hepatocellular carcinoma cell death induced by vorinostat via downregulating HIF-1 $\alpha$ under hypoxia. Biochem Biophys Res Commun 498: 481-486, 2018.

10. Huang GW, Yang LY and Lu WQ: Expression of hypoxia-inducible factor 1alpha and vascular endothelial growth factor in hepatocellular carcinoma: Impact on neovascularization and survival. World J Gastroenterol 11: 1705-1708, 2005.
11. Ellinghaus P, Heisler I, Unterschemmann K, Haerter M, Beck H, Greschat S, Ehrmann A, Summer H, Flamme I, Oehme F, et al: BAY 87-2243, a highly potent and selective inhibitor of hypoxia-induced gene activation has antitumor activities by inhibition of mitochondrial complex I. Cancer Med 2: 611-624, 2013.

12. Schöckel L, Glasauer A, Basit F, Bitschar K, Truong H, Erdmann G, Algire C, Hägebarth A, Willems PH, Kopitz C, et al: Targeting mitochondrial complex I using BAY 87-2243 reduces melanoma tumor growth. Cancer Metab 3: 11, 2015.

13. Basit F, van Oppen LM, Schöckel L, Bossenbroek HM, van Emst-de Vries SE, Hermeling JC, Grefte S, Kopitz C, Heroult M, Hgm Willems P and Koopman WJ: Mitochondrial complex I inhibition triggers a mitophagy-dependent ROS increase leading to necroptosis and ferroptosis in melanoma cells. Cell Death Dis 8: e2716, 2017.

14. Helbig L, Koi L, Brüchner K, Gurtner K, Hess-Stumpp H, Unterschemmann K, Baumann M, Zips D and Yaromina A: BAY 87-2243, a novel inhibitor of hypoxia-induced gene activation, improves local tumor control after fractionated irradiation in a schedule-dependent manner in head and neck human xenografts. Radiat Oncol 9: 207, 2014.

15. Häcker G: The morphology of apoptosis. Cell Tissue Res 301: 5-17, 2000.

16. Lan Y, Han J, Wang Y, Wang J, Yang G, Li K, Song R, Zheng T, Liang Y, Pan S, et al: STK17B promotes carcinogenesis and metastasis via AKT/GSK-3 $\beta /$ Snail signaling in hepatocellular carcinoma. Cell Death Dis 9: 236, 2018.

17. Liu L, Dai Y, Chen J, Zeng T, Li Y, Chen L, Zhu YH, Li J, Li Y, Ma S, et al: Maelstrom promotes hepatocellular carcinoma metastasis by inducing epithelial-mesenchymal transition by way of Akt/GSK-3 $\beta /$ Snail signaling. Hepatology 59: 531-543, 2014.

18. Peinado H, Portillo F and Cano A: Switching on-off Snail: LOXL2 versus GSK3beta. Cell Cycle 4: 1749-1752, 2005.

19. Cassim S, Raymond VA, Dehbidi-Assadzadeh L, Lapierre P and Bilodeau M: Metabolic reprogramming enables hepatocarcinoma cells to efficiently adapt and survive to a nutrient-restricted microenvironment. Cell Cycle 17: 903-916, 2018.

20. Amann T, Maegdefrau U, Hartmann A, Agaimy A, Marienhagen J, Weiss TS, Stoeltzing O, Warnecke C, Schölmerich J, Oefner PJ, et al: GLUT1 expression is increased in hepatocellular carcinoma and promotes tumorigenesis. Am J Pathol 174: 1544-1552, 2009.

21. Kitajima Y and Miyazaki K: The critical impact of HIF-1a on gastric cancer biology. Cancers (Basel) 5: 15-26, 2013.

22. Hall JL, Chatham JC, Eldar-Finkelman H and Gibbons GH: Upregulation of glucose metabolism during intimal lesion formation is coupled to the inhibition of vascular smooth muscle cell apoptosis. Role of GSK3beta. Diabetes 50: 1171-1179, 2001.

23. Zhou BP, Deng J, Xia W, Xu J, Li YM, Gunduz M and Hung MC: Dual regulation of Snail by GSK-3beta-mediated phosphorylation in control of epithelial-mesenchymal transition. Nat Cell Biol 6: 931-940, 2004.

24. Liu KY, Wang LT and Hsu SH: Modification of epigenetic histone acetylation in hepatocellular carcinoma. Cancers (Basel) 10: pii: E8, 2018

25. Lin KT, Wang YW, Chen CT, Ho CM, Su WH and Jou YS: HDAC inhibitors augmented cell migration and metastasis through induction of PKCs leading to identification of low toxicity modalities for combination cancer therapy. Clin Cancer Res 18: 4691-4701, 2012.

26. Xu W, Liu H, Liu ZG, Wang HS, Zhang F, Wang H, Zhang J, Chen JJ, Huang HJ, Tan Y, et al: Histone deacetylase inhibitors upregulate Snail via Smad2/3 phosphorylation and stabilization of Snail to promote metastasis of hepatoma cells. Cancer Lett 420: 1-13, 2018 